University of Nebraska - Lincoln

DigitalCommons@University of Nebraska - Lincoln

Mechanical \& Materials Engineering Faculty

Publications

Mechanical \& Materials Engineering,

Department of

6-14-2021

\title{
Minimally Invasive Delivery of 3D Shape Recoverable Constructs with Ordered Structures for Tissue Repair
}

\author{
Shixuan Chen \\ University of Nebraska Medical Center \\ Mark Alan Carlson \\ University of Nebraska Medical Center \\ Xiaowei Li \\ University of Nebraska Medical Center \\ Aleem Siddique \\ University of Nebraska Medical Center \\ Wuqiang Zhu \\ Center of Regenerative Medicine, Mayo Clinic
}

See next page for additional authors

Follow this and additional works at: https://digitalcommons.unl.edu/mechengfacpub

Part of the Mechanics of Materials Commons, Nanoscience and Nanotechnology Commons, Other

Engineering Science and Materials Commons, and the Other Mechanical Engineering Commons

Chen, Shixuan; Carlson, Mark Alan; Li, Xiaowei; Siddique, Aleem; Zhu, Wuqiang; and Xie, Jingwei, "Minimally Invasive Delivery of 3D Shape Recoverable Constructs with Ordered Structures for Tissue Repair" (2021). Mechanical \& Materials Engineering Faculty Publications. 613.

https://digitalcommons.unl.edu/mechengfacpub/613

This Article is brought to you for free and open access by the Mechanical \& Materials Engineering, Department of at DigitalCommons@University of Nebraska - Lincoln. It has been accepted for inclusion in Mechanical \& Materials Engineering Faculty Publications by an authorized administrator of DigitalCommons@University of Nebraska Lincoln. 


\section{Authors}

Shixuan Chen, Mark Alan Carlson, Xiaowei Li, Aleem Siddique, Wuqiang Zhu, and Jingwei Xie 


\title{
Minimally Invasive Delivery of 3D Shape Recoverable Constructs with Ordered Structures for Tissue Repair
}

\author{
Shixuan Chen ${ }^{\dagger}$, Mark Alan Carlson ${ }^{\ddagger}$, Xiaowei $\mathrm{Li}^{\Psi}$, Aleem Siddique ${ }^{\#}$, Wuqiang Zhu£ ${ }^{£}$ Jingwei \\ $\mathrm{Xie}^{\dagger, \S,{ }^{*}}$ \\ †Department of Surgery-Transplant and Mary \& Dick Holland Regenerative Medicine Program, \\ University of Nebraska Medical Center, Omaha, Nebraska 68198, United States \\ ‡Department of Surgery-General Surgery, University of Nebraska Medical Center, Omaha, \\ Nebraska 68198, United States \\ ${ }^{\Psi}$ Department of Neurological Sciences and Mary \& Dick Holland Regenerative Medicine \\ Program, College of Medicine, University of Nebraska Medical Center, Omaha, NE 68198, United \\ States \\ \#Department of Surgery-Cardiothoracic Surgery, College of Medicine, University of Nebraska \\ Medical Center, Omaha, NE 68198, United States \\ EDepartment of Cardiovascular Diseases, Physiology and Biomedical Engineering, Center of \\ Regenerative Medicine, Mayo Clinic, Scottsdale, AZ 85259, United States \\ $\S$ Department of Mechanical and Materials Engineering, University of Nebraska-Lincoln, Lincoln, \\ NE 68588, United States
}

\section{Abstract}

\begin{abstract}
Minimally invasive procedures are becoming increasingly more common in surgery. However, the biomaterials capable of delivering biomimetic, three-dimensional (3D) functional tissues in a minimally invasive manner and exhibiting ordered structures after delivery are lacking. Herein, we reported the fabrication of gelatin methacryloyl (GelMA)-coated, 3D expanded nanofiber scaffolds and their potential applications in minimally invasive delivery of 3D functional tissue constructs with ordered structures and clinically appropriate sizes $(4 \mathrm{~cm} \times 2 \mathrm{~cm} \times 1.5 \mathrm{~mm})$. GelMA-coated, expanded 3D nanofiber scaffolds produced by combining electrospinning, gas-foaming expansion, hydrogel coating, and crosslinking are extremely shape recoverable after release of compressive strain, displaying a superelastic property. Such scaffolds can be seeded with various types of cells, including dermal fibroblasts, bone marrow-derived mesenchymal stem cells, and human neural stem/precursor cells to form 3D complex tissue constructs. Importantly, the developed 3D tissue constructs can be compressed and loaded into a 4-mm diameter glass tube for minimally invasive delivery without compromising the cell viability. Taken together, the method developed in this
\end{abstract}

\footnotetext{
*Corresponding Authors jingwei.xie@unmc.edu., Phone: +1 (402) 5599442, Fax: +1(402) 5597521.

The authors declare no competing financial interest.

Supporting Information

Highly magnified SEM images of 3D expanded PCL nanofiber scaffolds without and with $0.05 \%$ GelMA coating; A SEM image of 3D expanded PCL nanofiber scaffolds with $0.1 \%$ GelMA coating; GFP labeled dermal fibroblast culture; Regions for confocal imaging
} 
study could hold great promise for transplantation of biomimetic, 3D functional tissue constructs with well-organized structures for tissue repair and regeneration using minimally invasive procedures like laparoscopy and thoracoscopy.

\section{Keywords}

expanded nanofiber scaffolds; stem cells; 3D tissue constructs; ordered structure; minimally invasive delivery

Based on tissue engineering and regenerative medicine principles, three-dimensional (3D) tissue constructs with a variety of functions and complex structures have been established for surgical transplantations to repair many types of tissues, often necessitating invasive surgical procedures. ${ }^{1-4}$ However, invasive surgical implantations are associated with an increased risk for perioperative and postoperative complications (e.g., infections). ${ }^{5}$

Researchers have attempted to develop other approaches for delivering cells in a minimally invasive manner (e.g., injection), including cell solutions, mixing cells with hydrogels, cellencapsulated hydrogel beads, cell-seeded microparticles/microspheres, cell aggregates/ organoids, cell microdroplets, and cell sheet fragments. ${ }^{6-15}$ However, these methods failed to supply 3D tissue constructs with specifically ordered structures and instantaneous functionality. Some of the approaches even led to low cell retention and survival rate and eventually failed to repair the targeted tissue defects after delivery. ${ }^{6,16}$ There is a great need to develop 3D functional tissue constructs with well-organized structures that can be delivered through minimally invasive procedures (e.g., without fully opening the chest).

Towards this end, several types of materials/scaffolds have been investigated for minimally invasive delivery of cells/tissues. Interconnected macroporous cryogels made of alginate, gelatin, hyaluronic acid (HA), HA-co-gelatin, and poly(ethylene glycol) diacrylate (PEGDA) with shape-memory properties were reported in many predefined geometries, which can be rehydrated with a suspension of cells (e.g., chondrocytes, mesenchymal stem cells (MSCs), fibroblasts, and neurons) and/or growth factors (e.g., vascular endothelial growth factor (VEGF)) and compressed into smaller forms and delivered through a small catheter or syringe injection for enhancing cell retention and revascularization. ${ }^{17-19}$ Porous collagen-coated carboxymethylcellulose microscaffolds seeded with OP9 mesenchymal cells and hematopoietic stem and progenitor cells (HSPCs) after in vitro co-culture were harvested as a paste-like living injectable niche through precisely controlled dehydration, preserving HSPC function. ${ }^{20}$ Flexible shape-memory poly(oxymethylene maleate (anhydride) citrate) scaffolds fabricated using a combination of conventional soft lithography and injection molding were seeded with cardiomyocytes to form cardiac patches $(1 \mathrm{~cm} \times 1 \mathrm{~cm} \times 0.1 \mathrm{~mm})$, which can regain their initial shape without influencing cell survival and function after delivery through a small orifice. ${ }^{21}$ Other than the shape-memory scaffolds, heart valve scaffolds made of polyglycolide acid mesh coated with poly-4hydroxybutyrate with seeding of myofibroblasts were delivered in a minimally invasive manner in combination with self-expandable stents. ${ }^{22}$ However, these scaffolds are associated with issues, including lack of extracellular matrix (ECM)-mimetic topography, absence of aligned nanotopographic cues, limited size and thickness (non-clinically relevant 
size and thickness), and/or necessitating the assistance from self-expandable stents. The aligned nanotopographic cues are essential for the repair of tissues (e.g., muscle, nerve, and tendon) with ordered structure. These tissues show an anisotropic characteristic of ECM topography. Aligned nanotopographic cues play an important role in regulating cell morphology and function and guiding ECM deposition. It is critical to use the constructs with ordered structure for repairing tissues like muscle, nerve, and tendon.

Here, we describe a new method for minimally invasive delivery of biomimetic, 3D functional tissue constructs with ordered structures with clinically relevant size and thickness for tissue repair as illustrated in Figure 1. The tissue constructs were built based on 3D expanded electrospun nanofiber scaffolds produced by our two recent vital findings. One is that innovative gas-foaming technology can expand 2D electrospun nanofiber mats to form 3D expanded scaffolds with layered structure and controlled alignment. ${ }^{24,25}$ Briefly, $\mathrm{NaBH}_{4}$ reacting with water produced $\mathrm{H}_{2}$ gas bubbles within nanofiber membranes. As the reaction proceeded, the $\mathrm{H}_{2}$ gas bubbles grew and/or merged, resulting in the expansion of nanofiber membranes. The other is that gelatin coating allows expanded nanofiber scaffolds to be superelastic, compressible, and shape recoverable. ${ }^{26}$ We hypothesized that $i$ ) stem cells (e.g., bone marrow-derived mesenchymal stem cells (BMSCs) and human neural stem/ progenitor cells (hNSCs)) could be seeded on 3D expanded nanofiber scaffolds and proliferate and/or differentiate throughout the scaffolds to form 3D tissue constructs; ii) the engineered 3D tissue constructs could be compressed and delivered to damaged/diseased tissue sites using minimally invasive surgical procedures such as laparoscopy and thoracoscopy; ${ }^{26,27}$ iii) the delivered tissue constructs can return to their original shape after delivery, and the shape-recovered 3D tissue constructs could effectively repair the damaged tissues (Figure 1).

To test our hypothesis, we first fabricated 3D expanded nanofiber scaffolds. Briefly, 1-mm thick poly ( $\varepsilon$-caprolactone) $(\mathrm{PCL})$ nanofiber stripes $(4 \mathrm{~cm} \times 1.5 \mathrm{~mm})$ were generated by electrospinning and cryocutting following our previous studies. ${ }^{28-30} \mathrm{Then}$, the stripes were expanded in the $\mathrm{NaBH}_{4}$ solution to form 2-cm wide scaffolds. The expanded 3D scaffolds were further coated with the $0.05 \%$ gelatin methacryloyl (GelMA) solution followed by crosslinking to enhance their mechanical properties and maintain the nanofibrous morphology. Figure 2a shows photographs of electrospun nanofiber stripes $(4 \mathrm{~cm} \times 1 \mathrm{~mm} \times$ $1.5 \mathrm{~mm}$ ). Figure $2 \mathrm{~b}$ shows the photographs of 3D expanded nanofiber scaffolds in different views, indicating the clinically relevant dimensions $(4 \mathrm{~cm} \times 2 \mathrm{~cm} \times 1.5 \mathrm{~mm})$ for a cardiac patch. ${ }^{31}$ Figure $2 \mathrm{c}$ shows scanning electron microscopy (SEM) images of expanded PCL nanofiber scaffolds, indicating the porous structure and alignment of fibers. Figure $2 \mathrm{~d}$ shows SEM images of expanded PCL nanofiber scaffolds after $0.05 \%$ GelMA coating and freezedrying. Intriguingly, part of the coated GelMA formed nanofibers throughout the expanded scaffold, resulting in a hybrid nanofiber scaffold (Figure S1). In contrast with PCL nanofibers, the GelMA nanofibers were randomly distributed and the diameter of GelMA nanofibers was not uniform (Figure S1). In contrast, a mass of GelMA sheets and small amount of GelMA nanofibers were seen throughout the expanded scaffold after $0.1 \%$ GelMA coating (Figure S2). The mechanism of GelMA nanofiber formation could be similar to the formation of nanofibers by freeze-drying dilute polymer solutions reported in previous studies as the GelMA concentration was $0.05 \% .{ }^{32}$ As described, during freeze- 
drying of a dilute solution, the polymer molecules could be excluded to form nanofibers because of the small number of polymer molecules available. In addition, a small amount of GelMA could be directly coated on the surface of PCL nanofibers without altering the original nanofibrous morphology. We then characterized the superelastic and shape recoverable properties of $0.05 \%$ GelMA coated, 3D expanded nanofiber scaffolds. Figure 3a shows that $0.05 \%$ GelMA coated, 3D expanded PCL nanofiber scaffolds can return to their original shapes after compression, pulling, rolling, and folding. The cyclic compression tests further confirmed their superelastic and shape recoverable properties. The 3D expanded nanofiber scaffolds without GelMA coating deformed after one time of compression in $90 \%$ compressive strain (Figure 3b). As expected, the GelMA-coated nanofiber scaffolds could return to their original shapes even after 100 times of compression with $90 \%$ compressive strain (Figure 3c). The GelMA coating significantly enhanced the compressive stress of 3D expanded nanofiber scaffolds in 50\%, 70\%, and $90 \%$ compressive strains, respectively (Figure 3d). Though the elastic modulus of GelMA-coated nanofiber scaffolds slightly decreased during the 100 cycles of cyclic compression in $90 \%$ compressive strain, the elastic modulus of GelMA-coated scaffolds were much higher than those of the scaffolds without coating at the $1^{\text {st }}, 25^{\text {th }}, 50^{\text {th }}, 75^{\text {th }}$, and $100^{\text {th }}$ cycle of cyclic compression (Figure $3 \mathrm{e}$ ). In addition, the GelMA-coated scaffold was able to recover its original shape in water after compression (Video S1). To understand the mechanism of superelastic and shape recoverable properties, we performed SEM imaging of cross-sections of 3D expanded nanofiber scaffolds without and with GelMA coating under compression in different compressive strains (Figure $3 \mathrm{f}$ and $3 \mathrm{~g}$ ). The cross-sections of 3D expanded nanofiber scaffolds with and without GelMA coating exhibited many arch-shaped pores. After compression in $50 \%, 70 \%$, and $90 \%$ compressive strains, the height of arches gradually decreased for both types of scaffolds. After release of compressive strain, the arches can almost return to their original shapes for GelMA-coated scaffolds (Figure 3g). In contrast, the arches in 3D expanded nanofiber scaffolds without GelMA coating failed to completely recover after strain release (Figure 3f). We speculate that the shape recoverable property could be due to the unique structure of 3D expanded nanofiber scaffolds that have hierarchically layered architectures forming numerous arches in micro-and millimeter scale. These arches act as elastic units and the GelMA coatings after crosslinking further reinforce the arches. Upon the compressive stress, the distortion of scaffolds is mainly attributed to the squeezing of the arches. The continuous compression causes the densification of arches. The top and bottom walls of arches eventually contact with each other and form the significant loading-bearing portion. The cross-linked GelMA coatings may help hold the nanofibers together at crossing points, nodes, and contacting surfaces, thus providing strong -fatigue resistance. Therefore, $0.05 \%$ GelMA coated nanofiber scaffolds can fully recover their shapes and retain their structural integrity in each compress-release cycle in different strains. Compared to $0.5 \%$ gelatin-coated nanofiber scaffold, $0.05 \%$ GelMA-coated nanofiber scaffolds showed a better superelastic property based on the cyclic compression test, ${ }^{25}$ which could be attributed to the intersecting hybrid nanofiber networks. In contrast, in $0.5 \%$ gelatin-coated nanofiber scaffolds, some pores may be blocked by the coated gelatin, resulting in the reduction of its superelastic property. 
We further cultured different types of cells on $0.05 \%$ GelMA-coated, 3D expanded nanofiber scaffolds $(2 \mathrm{~cm} \times 2 \mathrm{~cm} \times 1.5 \mathrm{~mm})$. Green fluorescence protein (GFP)-labeled dermal fibroblasts were first seeded on $0.05 \%$ GelMA-coated, 3D expanded nanofiber scaffolds. It is evident that the seeded and proliferated fibroblasts were elongated along the direction of nanofiber alignment after incubation for 1, 3, 7, and 14 days (Figure S3a). Besides, the seeded fibroblasts proliferated rapidly on the nanofiber scaffolds from day 1 to day 14 as indicated by cell densities contributed by the fluorescence signals and the quantified CCK-8 assay (Figure S3a and Figure S3b). The cells entirely covered the scaffolds after 14 days of culture (Figure S3c). BMSCs have been used in repairing and regenerating many tissue types. ${ }^{33,34}$ We then demonstrated the attachment and proliferation of BMSCs on $0.05 \%$ GelMA-coated, 3D expanded nanofiber scaffolds. Figure 4a shows confocal microscopy images of the BMSCs throughout the four regions of scaffolds labeled in Figure S4 after 14 days of incubation. The cell F-actin was stained with Alexa Fluor ${ }^{\mathrm{TM}} 546$ Phalloidin in red, and the cell nucleus was stained with 4',6-diamidino-2-phenylindole (DAPI) in blue (Figure 4a). In addition, hNSCs have shown potential in treatment of disease or injuries of the central and peripheral nervous systems. ${ }^{35,36}$ We then demonstrated the proliferation and differentiation of hNSCs on $0.05 \%$ GelMA-coated, 3D expanded nanofiber scaffolds. The hNSCs were able to cover most of the area of the scaffolds after 7 days of proliferation and 14 days of differentiation (Figure $4 \mathrm{~b}$ ). Most neurites extended along the direction of nanofiber alignment. Some neurites may cross through the nanofiber layers (Figure 4b).

We finally examined the effect of minimally invasive delivery on the viability of cells in 3D shape-recoverable tissue constructs. BMSCs were seeded on 0.05\% GelMA-coated, 3D expanded nanofiber scaffolds $(4 \mathrm{~cm} \times 2 \mathrm{~cm} \times 1.5 \mathrm{~mm})$. These cells proliferated for 14 days to form $3 \mathrm{D}$ tissue constructs which were then compressed and loaded into a glass tube (diameter $=4 \mathrm{~mm}$; length $=15 \mathrm{~cm}$ ) with assistant of sharp tweezers (Figure 5a). One end of the glass tube was connected to a filtered air source, and the compressed, BMSCs-seeded scaffold inside the glass tube was blown into a culture dish containing medium and reexpanded (Figure 5a and Video S2). No significant difference was observed between the cell viabilities of BMSCs on scaffolds before and after delivery (Figure 5b and Figure 5c). Similarly, the hNSCs-seeded nanofiber scaffolds can be delivered through the same method (Video S3). Alternatively, culture medium or saline flowing through the glass tube was able to push the compressed, cell-seeded scaffolds to the targeted tissue as well (Video S4).

In summary, we have demonstrated the fabrication of $0.05 \%$ GelMA-coated, 3D expanded nanofiber scaffolds. Such scaffolds were superelastic and shape recoverable. These scaffolds after seeding with different cell types can form 3D tissue constructs with ordered structure. Importantly, these 3D tissue constructs can be delivered via a minimally invasive approach without compromising the viability of seeded cells. Future studies will be focused on the investigation of the efficacy of 3D tissue constructs consisting of cells and $0.05 \%$ GelMAcoated, 3D expanded nanofiber scaffolds in relevant large animal models. ${ }^{31}$ In addition, signaling molecules can be further incorporated to the nanofiber scaffolds to regulate cell response and enhance tissue repair. ${ }^{30} 3 \mathrm{D}$ bioprinting could be used to pattern cells throughout the shape-recoverable nanofiber scaffolds. ${ }^{37}$ The strategy presented in this study holds great promise for repairing and regenerating tissues (e.g., heart) using minimally invasive surgical procedures. 


\section{Materials and Methods.}

\section{Materials.}

PCL (Mw= $80 \mathrm{kDa}$ ), pluronic-F-127, gelatin, sodium borohydride, Triton X-100 were purchased from Sigma-Aldrich (St. Louis, MO, USA). Dichloromethane (DCM) and N, Ndimethylformamide (DMF) were purchased from BDH Chemicals (Dawsonville, GA, USA). Dulbecco's modified eagle medium (DMEM), fetal bovine serum (FBS), and penicillinstreptomycin were obtained from Invitrogen (Carlsbad, CA, USA). Basic fibroblast growth factor (FGF-2), epidermal growth factor (EGF), brain-derived neurotrophic factor (BDNF), glial cell-derived neurotrophic factor (GDNF) were obtained from Peptrotech (Rocky Hill, NJ, USA). The ReNcell Neural Stem Cell Medium was purchased from Millipore (Billerica, MA, USA). Tuj1 primary antibody and laminin were obtained from Millipore Sigma (Burlington, MA, USA). Goat anti-mouse IgG H\&L (Alexa Fluor® 546) secondary antibody, Alexa Fluor ${ }^{\mathrm{TM}} 546$ Phalloidin, DAPI were ordered from Abcam (Cambridge, MA, USA).

\section{Fabrication of $\mathbf{0 . 0 5 \%}$ GelMA coated, 3D expanded nanofiber scaffolds.}

The PCL/0.5\% F-127 nanofiber mat was prepared following our previous study. ${ }^{28-30}$ The nanofiber mat (thickness $=1 \mathrm{~mm}$ ) was immersed in liquid nitrogen and then cut into stripes $(4 \mathrm{~cm} \times 1.5 \mathrm{~mm} \times 1 \mathrm{~mm})$. The nanofiber stripes were subsequently immersed into the $1 \mathrm{M}$ $\mathrm{NaBH}_{4}$ solution and treated with vacuum for $3 \mathrm{~s}$. The fiber mats were continuously expanded in the $1 \mathrm{M} \mathrm{NaBH}_{4}$ solution for another $30 \mathrm{~s}$ at ambient condition. The expanded nanofiber scaffolds were placed into a 3D printed mold $(4 \mathrm{~cm} \times 2 \mathrm{~cm} \times 1.5 \mathrm{~mm})$, treated with vacuum until they froze, and dried by freeze-drying. For GelMA coating, the freezedried expanded nanofiber scaffolds were immersed in the $0.05 \%$ GelMA solution plus with $0.1 \% 2959$ photocrosslinker which was added into the mold. Then, the scaffolds were crosslinked with UV light for $45 \mathrm{~s}$ and dried by freeze-drying.

\section{SEM characterization.}

The 3D expanded nanofiber scaffolds with or without $0.05 \%$ GelMA coating were coated with Pt for 5 min using a Pt sputter coater and imaged by SEM under 25 kV (FEI, Quanta 200, Hillsboro, OR, USA). Aluminum sheets confined the nanofiber scaffolds in different compressive strains during the SEM imaging.

\section{Cyclic compression tests.}

The mechanical properties of 3D expanded nanofiber scaffolds with or without $0.05 \%$ GelMA coating were measured by an Instron 5640 universal test machine. Samples $(10 \mathrm{~mm}$ $\times 10 \mathrm{~mm} \times 10 \mathrm{~mm}$ ) were used to perform the cyclic compressive tests. The compressive strains were set as 50\%, 70\%, and 90\%, respectively. The mechanical tests were run 1 cycle with a speed of $9 \mathrm{~mm} / \mathrm{min}$. The superelastic property of $0.05 \%$ GelMA-coated, 3D expanded nanofiber scaffolds were run 100 cycles with a speed of $9 \mathrm{~mm} / \mathrm{min}$ under $90 \%$ compressive strain. The elastic modulus was obtained based on the initial slope before $30 \%$ of compressive strain. The recovery rate was calculated using the following equation. Recovery rate $(\%)=($ pore size after expansion / pore size before expansion $) \times 100 \%$ 


\section{Fibroblasts culture.}

The GFP-labeled human dermal fibroblasts were maintained in the low glucose DMEM medium containing $10 \%$ FBS and $1 \%$ penicillin-streptomycin. Twenty milliliters of fibroblast suspension solution $\left(1 \times 10^{7}\right.$ cells $\left./ \mathrm{ml}\right)$ was first prepared. The $0.05 \%$ GelMAcoated, 3D expanded nanofiber scaffolds were put into the cell suspension solution and treated with vacuum for $10 \mathrm{~s}$. These nanofiber scaffolds were then removed from the cell solution and placed into a $0.1 \%$ agar pretreated 6-well plate. The cell-seeded nanofiber scaffolds were continuously cultured for 1, 3, 7, and 14 days. At each indicated time point, a part of the scaffold $(5 \mathrm{~mm} \times 5 \mathrm{~mm})$ was cut by scissors, then it was put into a 24 -well culture plate, added $20 \mu \mathrm{l} \mathrm{CCK}-8$ and continuously cultured for $3 \mathrm{~h}$. The absorbance value at 450 $\mathrm{nm}$ was measured by a microplate reader.

\section{BMSCs culture.}

The rat BMSCs were isolated based on our established protocols and maintained in the low glucose DMEM medium containing $10 \%$ FBS and $1 \%$ penicillin-streptomycin. ${ }^{30}$ Twenty milliliters of BMSCs suspension solution $\left(1 \times 10^{7} \mathrm{cells} / \mathrm{ml}\right)$ was prepared. The $0.05 \%$ GelMA-coated, 3D expanded nanofiber scaffolds were put into the cell suspension solution and treated with vacuum for $10 \mathrm{~s}$. These nanofiber patch scaffolds were then removed from the cell solution and placed into a $0.1 \%$ agar pretreated 6-well plate. The nanofiber patch scaffolds were continuously cultured for 1, 3, 7, and 14 days respectively.

\section{Human NSC culture.}

Human NSCs were obtained from Millipore (Billerica, MA, USA). Laminin $(20 \mu \mathrm{g} / \mathrm{ml}$, Invitrogen) was used to coat tissue culture plastic-ware at least $4 \mathrm{~h}$ before cell seeding to promote the attachment of hNSCs. Cells were maintained in ReNcell Neural Stem Cell Medium (Millipore) with FGF-2 $(20 \mathrm{ng} / \mathrm{ml})$ and EGF $(20 \mathrm{ng} / \mathrm{ml})$ at $37{ }^{\circ} \mathrm{C}$ under $5 \% \mathrm{CO}_{2}$. Then, $20 \mathrm{ml}$ of hNSCs suspension with a concentration of $1 \times 10^{7}$ cell $/ \mathrm{ml}$ were first prepared. The $0.05 \%$ GelMA-coated, 3D expanded nanofiber scaffolds were immersed in the cell suspension solution and treated with a vacuum for $10 \mathrm{~s}$. The nanofiber scaffolds were then removed from the cell solution and placed into a $0.1 \%$ agar pretreated 6-well plate, and continuously cultured for 1 week. The culture medium was changed every two days. Then, hNSCs were cultured in the medium in the presence of BDNF $(20 \mathrm{ng} / \mathrm{ml})$ and GDNF (20 $\mathrm{ng} / \mathrm{ml}$ ) for neuronal differentiation for another two weeks.

\section{Confocal imaging.}

The GFP-labeled dermal fibroblasts seeded nanofiber scaffolds were directly imaged by confocal laser scanning microscopy (CLSM) (Zeiss 880, Oberkochen, Germany) after fixation with $4 \%$ paraformaldehyde. The BMSCs seeded nanofiber scaffolds were fixed with $4 \%$ paraformaldehyde and permeabilized with a $0.1 \%$ Triton X-100 solution. Subsequently, the BMSCs were stained with Alexa Fluor ${ }^{\mathrm{TM}} 546$ Phalloidin (dilution 1:200) for 20 min and DAPI (dilution 1:2000) for $5 \mathrm{~min}$. Then, the BMSCs seeded nanofiber scaffolds were imaged by confocal microscopy. The hNSCs seeded nanofiber scaffolds were fixed with $4 \%$ paraformaldehyde and permeabilized with $0.1 \%$ Triton X-100 in PBS solution for 20 min. Next, the whole scaffolds were blocked with $5 \%$ goat serum for $30 \mathrm{~min}$. The cells were then 
incubated with Tuj1 (1:100) primary antibody overnight, followed by incubation with goat anti-mouse IgG H\&L (Alexa Fluor® 546) secondary antibody (dilution $1: 200$ ) for $1 \mathrm{~h}$ and DAPI (dilution 1:2000) for $5 \mathrm{~min}$. Finally, cells-seeded scaffolds were imaged by confocal microscopy. The confocal microscopy imaging parameters were set as follows. The z-stack range was set from $0 \mu \mathrm{m}$ to $125 \mu \mathrm{m}$. The interval was set at $5 \mu \mathrm{m}$. The tile scans were set as $7 \times 7$ for one region of the scaffold, and four regions of the scaffolds were imaged as shown in Figure $\mathrm{S} 4$.

\section{Demonstration of minimally invasive delivery.}

The glass tube for minimally invasive delivery was modified from Wilmad ${ }^{\circledR}$ NMR tube (inner diameter $=4 \mathrm{~mm})$. The BMSCs and hNSCs seeded nanofiber scaffolds $(4 \mathrm{~cm} \times 2 \mathrm{~cm}$ $\times 1.5 \mathrm{~mm}$ ) were compressed and loaded into the glass tube. Then, one end of the tube was connected to an air source. The BMSCs and hNSCs seeded nanofiber scaffolds were pushed into a culture dish containing medium. The viability of BMSCs and hNSCs before and after delivery were evaluated by LIVE/DEAD assay. The BMSCs and hNSCs seeded nanofiber scaffolds were incubated with LIVE/DEAD assay kit for $10 \mathrm{~min}$, and then the scaffolds were imaged under the confocal microscope. The cells stained with red color (dead cells) and all cells in each image were counted, the apoptotic ratio of cells was calculated as following, the apoptotic ratio $(\%)=$ (number of dead cells / number of all cells) $\%$. Five randomly selected images were used to quantify the apoptotic ratio.

\section{Statistical analysis.}

All data were presented as mean \pm standard deviation. The statistical analysis was performed using GraphPad Prism 8.0 software. Differences between the two groups were evaluated using Student's t-test. The values of $p<0.05$ were considered statistically significant. The values of $\mathrm{p}<0.01$ were considered statistically very significant.

\section{Supplementary Material}

Refer to Web version on PubMed Central for supplementary material.

\section{ACKNOWLEDGMENTS}

This work was partially supported by startup funds from the University of Nebraska Medical Center (UNMC), National Institute of General Medical Science (NIGMS) of the National Institutes of Health under Award Numbers R01GM123081 and R01GM138552, Congressionally Directed Medical Research Program (CDMRP)/Peer Reviewed Medical Research Program (PRMRP) FY19 W81XWH2010207, and Nebraska Research Initiative grant.

\section{REFERENCES}

(1). Vacanti JP; Langer R Tissue Engineering: the Design and Fabrication of Living Replacement Devices for Surgical Reconstruction and Transplantation. Lancet 1999, 354, 32.

(2). Langer R; Vacanti J Advances in Tissue Engineering. J. Pediatr. Surg 2016, 51, 8. [PubMed: 26711689]

(3). Shafiee A; Atala A Tissue Engineering: Toward a New Era of Medicine. Ann. Rev. Med 2017, 68, 29. [PubMed: 27732788]

(4). Khademhosseini A; Langer R A Decade of Progress in Tissue Engineering. Nat. Protocol 2016, $11,1775$. 
(5). Darouiche RO Treatment of Infections Associated with Surgical Implants. N. Engl. J. Med 2004, 350, 1422. [PubMed: 15070792]

(6). Amer MH; Rose FRA; Shakesheff KM; Modo M; White LJ Translational Considerations in Injectable Cell-Based Therapeutics for Neurological Applications: Concepts, Progress and Challenges. npj Reg. Med 2017, 2, 23.

(7). Park SH; Seo JY; Park JY; Ji YB; Kim K; Choi HS; Choi S; Kim J; Min BH; Kim MS An Injectable, Click-crosslinked, Cytomodulin-Modified Hyaluronic Acid Hydrogel for Cartilage Tissue Engineering. NPG Asia Mater. 2019, 11, 30.

(8). Mao AS; Ozakle B; Shah NJ; Vining KH; Descombes T; Zhang L; Tringides CM; Wong SW; Shin JW; Scadden DT; Weitz DA; Mooney DJ Programmable Microencapsulation for Enhanced Mesenchymal Stem Cell Persistence and Immunomodulation. Proc. Natl. Acad. Sci. USA 2019, 116, 15392. [PubMed: 31311862]

(9). Matta R; Lee S; Genet N; Hirschi KK; Thomas JL; Gonzalez AJ Minimally Invasive Delivery of Microbeads with Encapsulated, Viable and Quiescent Neural Stem Cells to the Adult Subventricular Zone. Sci. Rep 2019, 9, 17798. [PubMed: 31780709]

(10). John JV; McCarthy A; Wang H; Chen S; Su Y; Davis E; Li X; Park JS; Reinhardt RA; Xie J Engineering Biomimetic Nanofiber Microspheres with Tailored Size, Predesigned Structure, and Desired Composition via Gas Bubble-Mediated Coaxial Electrospray. Small 2020, 16, 1907393.

(11). Kankala RK; Zhao J; Liu CG; Song XJ; Yang DY; Zhu K; Wang SB; Zhang YS; Chen AZ Highly Porous Microcarriers for Minimally Invasive in situ Skeletal Muscle Cell Delivery. Small 2019, 15, e1901397. [PubMed: 31066236]

(12). Zhao S; Xu Z; Wang H; Reese BE; Gushchina LV; Jiang M; Agarwal P; Xu J; Zhang M; Shen R; Liu Z; Weisleder N; He X Bioengineering of Injectable Encapsulated Aggregates of Pluripotent Stem Cells for Therapy of Myocardial Infarction. Nat. Commun 2016, 7, 13306. [PubMed: 27786170]

(13). Rossen NS; Anandakumaran PN; Nieden RZ; Lo K; Luo W; Park C; Huyan C; Fu Q; Song Z; Singh-Moon RP; Chung J; Goldenberg JE; Sampat N; Harimoto T; Bajakian DR; Gillette BM; Sia SK Injectable Therapeutic Organoids using Sacrificial Hydrogels. iScience 2020, 22, 101052.

(14). Gal I; Edri R; Noor N; Rotenberg M; Namestnikov M; Cabilly I; Shapira A; Dvir T Injectable Cardiac Cell Microdroplets for Tissue Regeneration. Small 2020, 16, 1904806.

(15). Wang CC; Chen CH; Lin WW; Hwang SM; Hsieh PCH; Lai PH; Yeh YC; Chang Y; Sung HW Direct Intramyocardial Injection of Mesenchymal Stem Cell Sheet Fragments Improves Cardiac Functions after Infarction. Cardiovasc. Res 2008, 77, 515. [PubMed: 18006453]

(16). Terrovitis JV; Smith RR; Marban E Assessment and Optimization of Cell Engraftment after Transplantation into the Heart. Cir. Res 2010, 106. 479.

(17). Bencherif SA; Sands RW; Bhatta D; Arany P; Verbeke CS; Edwards DA; Mooney DJ Injectable Preformed Scaffolds with Shape-Memory Properties. Proc. Natl. Acad. Sci. USA 2012, 109, 19590. [PubMed: 23150549]

(18). Memic A; Colombani T; Eggermont LJ; Rezaeeyazdi M; Steingold J; Rogers ZJ; Navare KJ; Mohammed HS; Benherif SA Latest Advances in Cryogel Technology for Biomedical Applications. Adv. Ther 2019, 2, 1800114.

(19). Eggermont LJ; Rogers ZJ; Colombani T; Memic A; Bencherif SA Injectable Cryogels for Biomedical Applications. Trends Biotechnol. 2020, 38, 418. [PubMed: 31699534]

(20). Tavakol DN; Tratwal J; Bonini D; Genta M; Campos V; Burch P; Hoehnel S; Beduer A; Alessandrini M; Naveiras O; Braschler T Injectable, Scalable 3D Tissue-engineered Model of Marrow Hematopoiesis. Biomaterials 2020, 232, 119665. [PubMed: 31881380]

(21). Montgomery M; Ahadian S; Huyer LD; Rito ML; Civitarese RA; Vanderlaan RD; Wu J; Reis LA; Momen A; Akbari S; Pahnke A; Li RK; Caldarone CA; Radisic M Flexible Shape-memory Scaffold for Minimally Invasive Delivery of Functional Tissues. Nat. Mater 2017, 16, 1038. [PubMed: 28805824]

(22). Schmidt D; Dijkman PE; Driessen-Mol A; Stenger R; Mariani C; Puolakka A; Rissanen M; Deichmann T; Odermatt B; Weber B; Emmert MY; Zund G; Baaijens FPT; Hoerstrup SP Minimally-Invasive Implantation of Living Tissue Engineered Heart Valves: a Comprehensive 
Approach from Autologous Vascular Cells to Stem Cells. J. Am. Coll. Cardiol 2010, 56, 510. [PubMed: 20670763]

(23). Jiang J; Carlson MA; Teusink MJ; Wang H; MacEwan MR; Xie J Expanding Two-dimensional Electrospun Nanofiber Membranes in the Third Dimension by a Modified Gas-foaming Technique. ACS Biomater. Sci. Eng 2015, 1, 991. [PubMed: 33429530]

(24). Jiang J; Li Z; Wang H; Wang Y; Carlson MA; Teusink MJ; MacEwan MR; Gu Li.; Xie J Expanded 3D Nanofiber Scaffolds: Cell Penetration, Neovascularization, and Host Response. Adv. Healthc. Mater 2016, 5, 2993. [PubMed: 27709840]

(25). Chen S; Carlson MA; Zhang YS; Hu Y; Xie J Fabrication of Injectable and Superelastic Nanofiber Rectangle Matrices ("Peanuts") and Their Potential Applications in Hemostasis. Biomaterials 2018, 179, 46. [PubMed: 29980074]

(26). Fuchs KH Minimally Invasive Surgery. Endoscopy 2002, 34, 154. [PubMed: 11822011]

(27). Robinson TN; Stiegmann GV Minimally Invasive Surgery. Endoscopy 2004, 36, 48. [PubMed: 14722855]

(28). Chen S; Wang H; McCarthy A; Yan Z; Kim HJ; Carlson MA; Xia Y; Xie J Three-dimensional Objects Consisting of Hierarchically Assembled Nanofibers with Controlled Alignments for Regenerative Medicine. Nano Lett. 2019, 19, 2059. [PubMed: 30788971]

(29). Chen S; John JV; McCarthy A; Carlson MA; Li X; Xie J Fast Transformation of 2D Nanofiber Membranes into Pre-molded 3D Scaffolds with Biomimetic and Oriented Porous Structure for Biomedical Applications. Appl. Phys. Rev 2020, 7, 021406. [PubMed: 32494338]

(30). Chen S; McCarthy A; John JV; Su Y; Xie J Converting 2D nanofiber membranes to 3D Hierarchical Assemblies with Structural and Compositional Gradients Regulates Cell Behavior. Adv. Mater 2020, 32, 2003754.

(31). Gao L; Gregorich ZR; Zhu W; Mattapally S; Oduk Y; Lou X; Kannappan R; Borovjagin AV; Walcott GP; Pollard AE; Fast VG; Hu X; Lloyd SG; Ge Y; Zhang J Large Cardiac-muscle Patches Engineered from Human Induced-Pluripotent Stem-Cell-Derived Cardiac Cells Improve Recovery from Myocardial Infarction in Swine. Circulation 2018, 137, 1712. [PubMed: 29233823]

(32). Qian L; Willneff E; Zhang H A Novel Route to Polymeric Sub-micron Fibers and Their Use as Templates for Inorganic Structures. Chem. Commun 2009, 26, 3946.

(33). Pittenger MF; Discher DE; Peault BM; Phinney DG; Hare JM; Caplan AI Mesenchymal Stem Cell Perspective: Cell Biology to Clinical Progress. npj Reg. Med 2019, 4, 22.

(34). Golpanian S; Wolf A; Hatzistergos KE; Hare JM Rebuilding the Damaged Heart: Mesenchymal Stem Cells, Cell-Based Therapy, and Engineered Heart Tissue. Phys. Rev 2016, 96, 1127.

(35). Yi S; Zhang Y; Gu X; Huang L; Zhang K; Qian T; Gu X Application of Stem Cells in Peripheral Nerve Regeneration. Burns Trauma 2020, 8, tkaa002. [PubMed: 32346538]

(36). Rosenzweig ES; Brock JH; Lu P; Kumamaru H; Salegio EA; Kadoya K; Weber JL; Liang JJ; Moseanko R; Hawbecker S; Huie JR; Havton LA; Nout-Lomas YS; Ferguson AR; Beattie MS; Bresnahan JC; Tuszynski MH Restorative Effects of Human Neural Stem Cell Grafts on the Primate Spinal Cord. Nat. Med 2018, 24, 484. [PubMed: 29480894]

(37). Murphy SV; Atala A 3D Bioprinting of Tissues and Organs. Nat. Biotech 2014, 32, 773-785. 


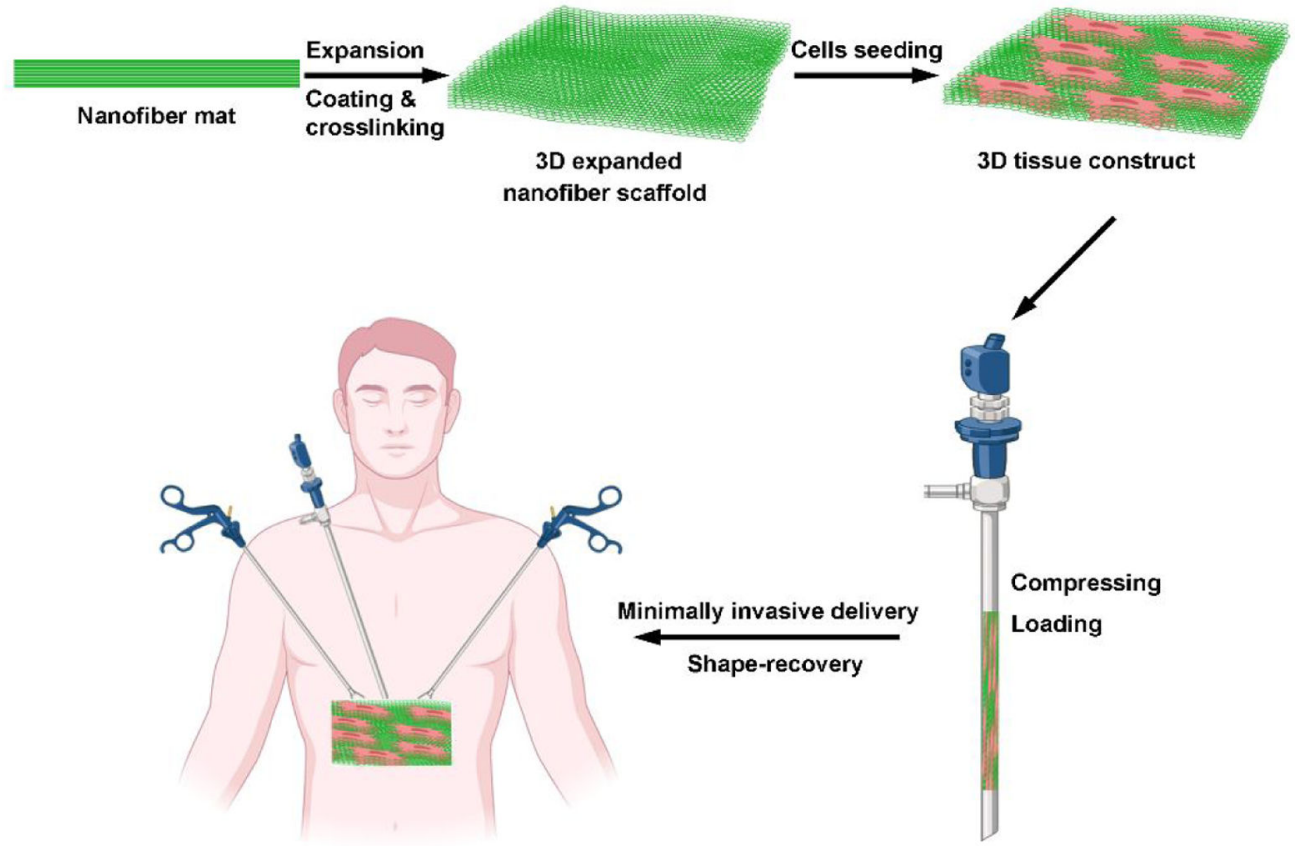

Figure 1.

Schematic illustrating the fabrication of shape-recoverable 3D tissue constructs, and their minimally invasive delivery. Procedures of fabrication of shape-recoverable 3D tissue constructs and their minimally invasive delivery include generation of electrospun nanofiber mat, expansion of nanofiber mat, GelMA coating, crosslinking, cell seeding, compression of 3D tissue constructs, loading into a cannula, injection, and shape-recovery. 
a

Before expansion

b

After expansion

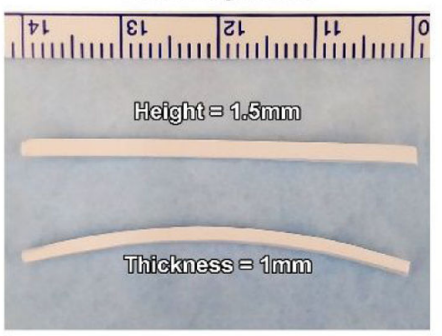

C Without coating
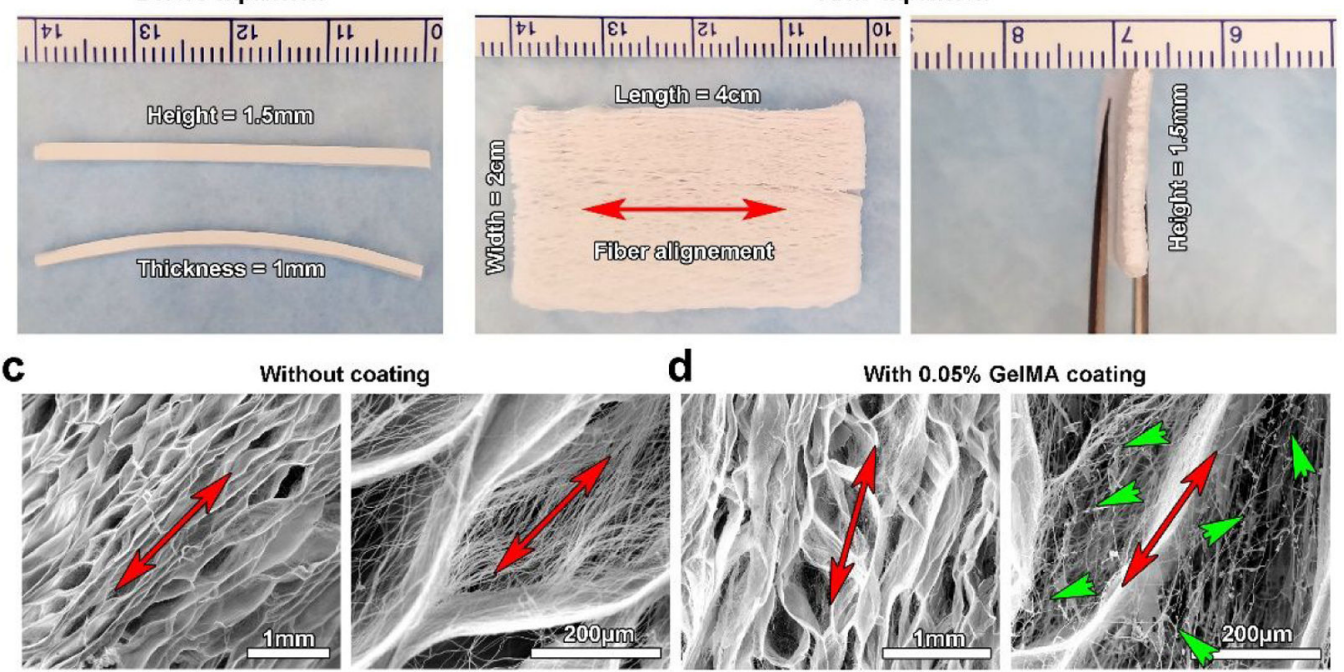

d With $0.05 \%$ GelMA coating
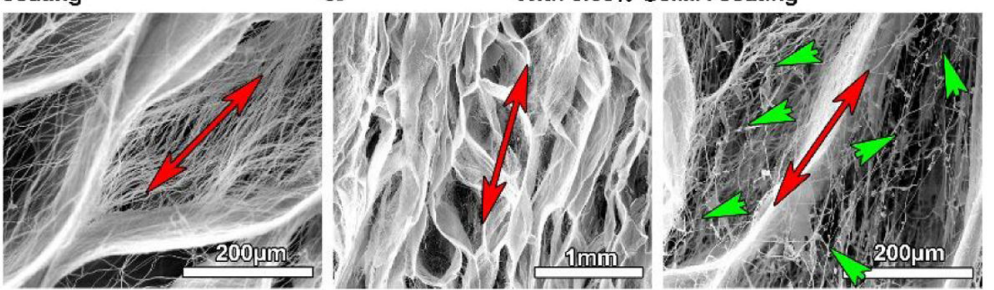

Figure 2.

3D expanded nanofiber scaffold fabrication and characterization. (a) Photograph showing PCL nanofiber stripes $(4 \mathrm{~cm} \times 1.5 \mathrm{~mm} \times 1 \mathrm{~mm})$ before expansion. (b) Photograph showing a 3D expanded PCL nanofiber scaffold $(4 \mathrm{~cm} \times 1.5 \mathrm{~mm} \times 2 \mathrm{~cm})$. (c) SEM images showing 3D expanded PCL nanofiber scaffolds without $0.05 \%$ GelMA coating, indicating the porous and layered structure. (d) SEM images showing 3D expanded PCL nanofiber scaffolds with $0.05 \%$ GelMA coating, indicating the formation of hybrid nanofiber scaffolds with a porous and layered structure. Red arrows indicate the direction of fiber alignment. Green arrow heads indicate the formation of GelMA nanofibers. 
a

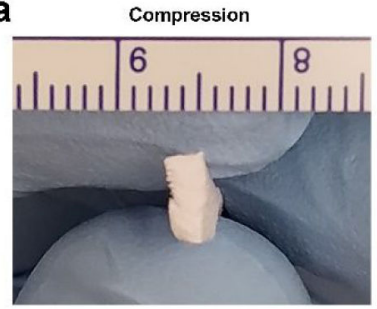

b

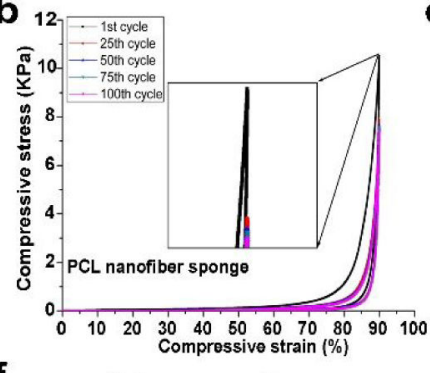

f

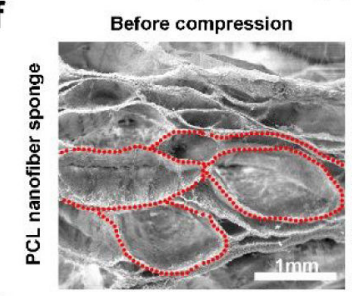

Before compression

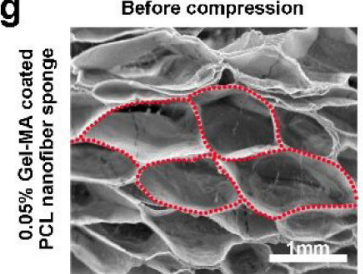

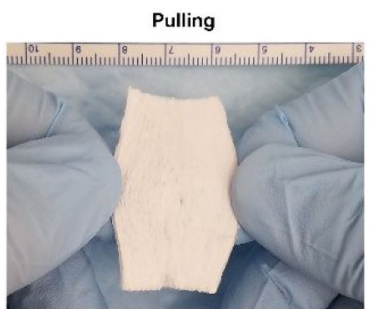

C

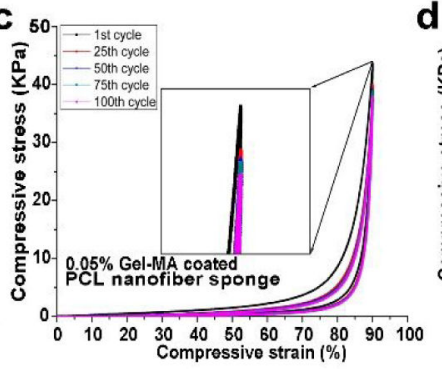

$50 \%$

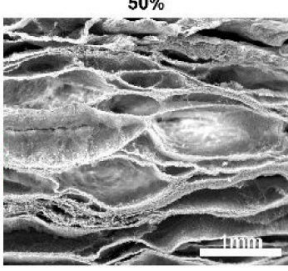

$50 \%$

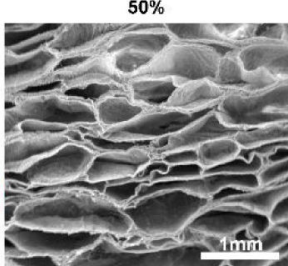

d

$70 \%$

$70 \%$
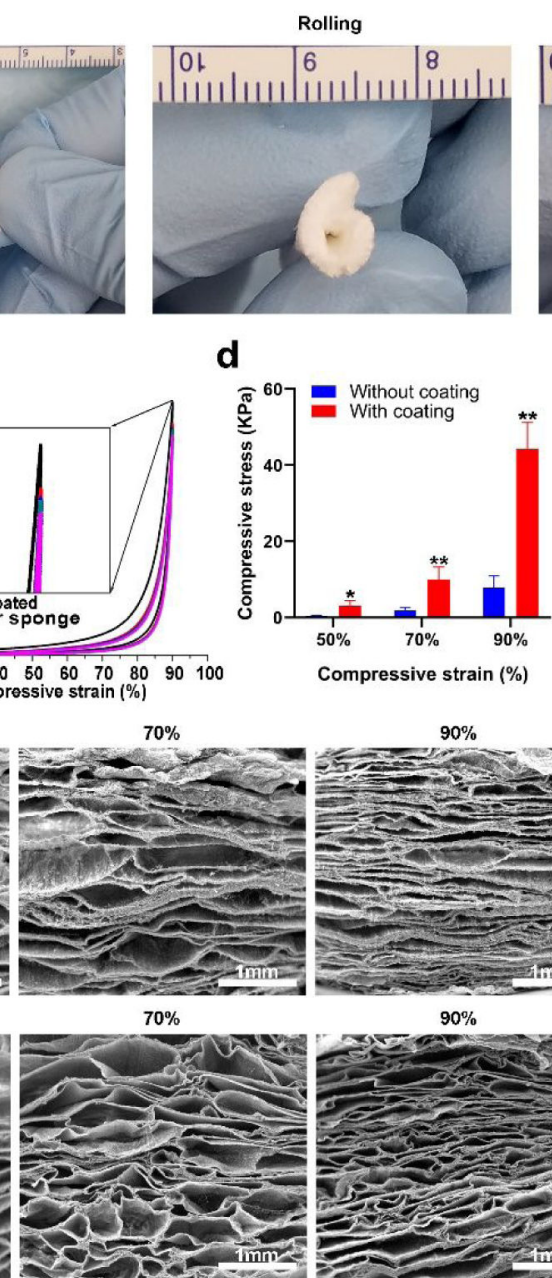

$90 \%$

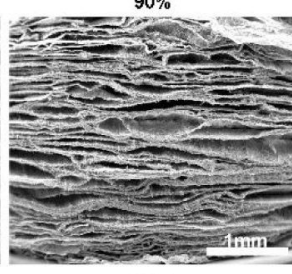

$90 \%$

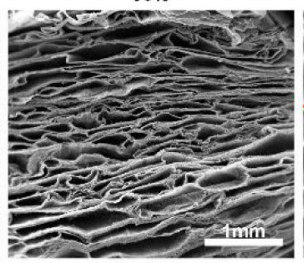

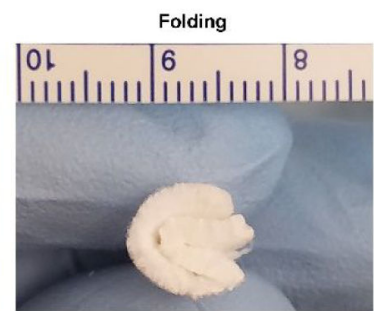

e

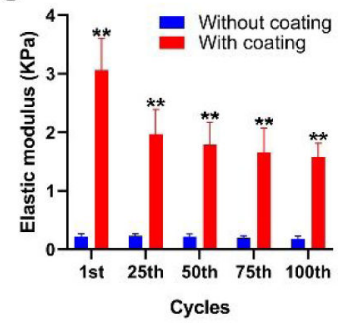

After strain release

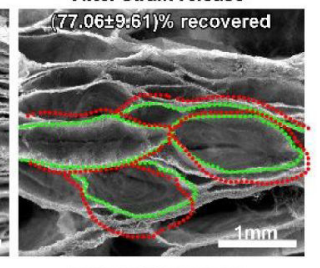

After strain release

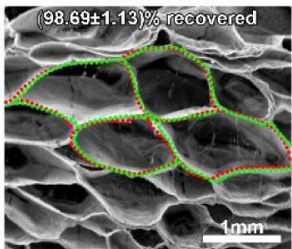

Figure 3.

The superelastic and shape recoverable properties of $0.05 \%$ GelMA-coated, 3D expanded PCL nanofiber scaffolds. (a) Photographs showing the flexible properties of $0.05 \%$ GelMAcoated, 3D expanded PCL nanofiber scaffolds. Cyclic compressive tests of 3D expanded, PCL nanofiber scaffolds without (b) and with (c) $0.05 \%$ GelMA coating in $90 \%$ compressive strain (100 cycles). (d) The maximum compressive stress of 3D expanded, PCL nanofiber scaffolds without and with $0.05 \%$ GelMA coating in 50\%, 70\%, and 90\% compressive strain. (e) The elastic modulus of 3D expanded, PCL nanofiber scaffolds without and with $0.05 \%$ GelMA coating after the $1^{\text {st }}, 25^{\text {th }}, 50^{\text {th }}, 75^{\text {th }}$, and $100^{\text {th }}$ cyclic compression. SEM images showing the corresponding 3D expanded, PCL nanofiber scaffolds without (f) and with $0.05 \%$ GelMA coating (g) before compression, in different compressive strains, and after release of compressive strains. 3D expanded, PCL nanofiber scaffolds without $0.05 \%$ GelMA coating failed to completely recover the shape after release of compressive strains. 3D expanded PCL nanofiber scaffolds with $0.05 \%$ GelMA coating could fully recover after release of compressive strains. Red dot lines indicate the contours of pores before compression. Green dot lines indicate the original shapes of pores after compression and release of compressive strain. 

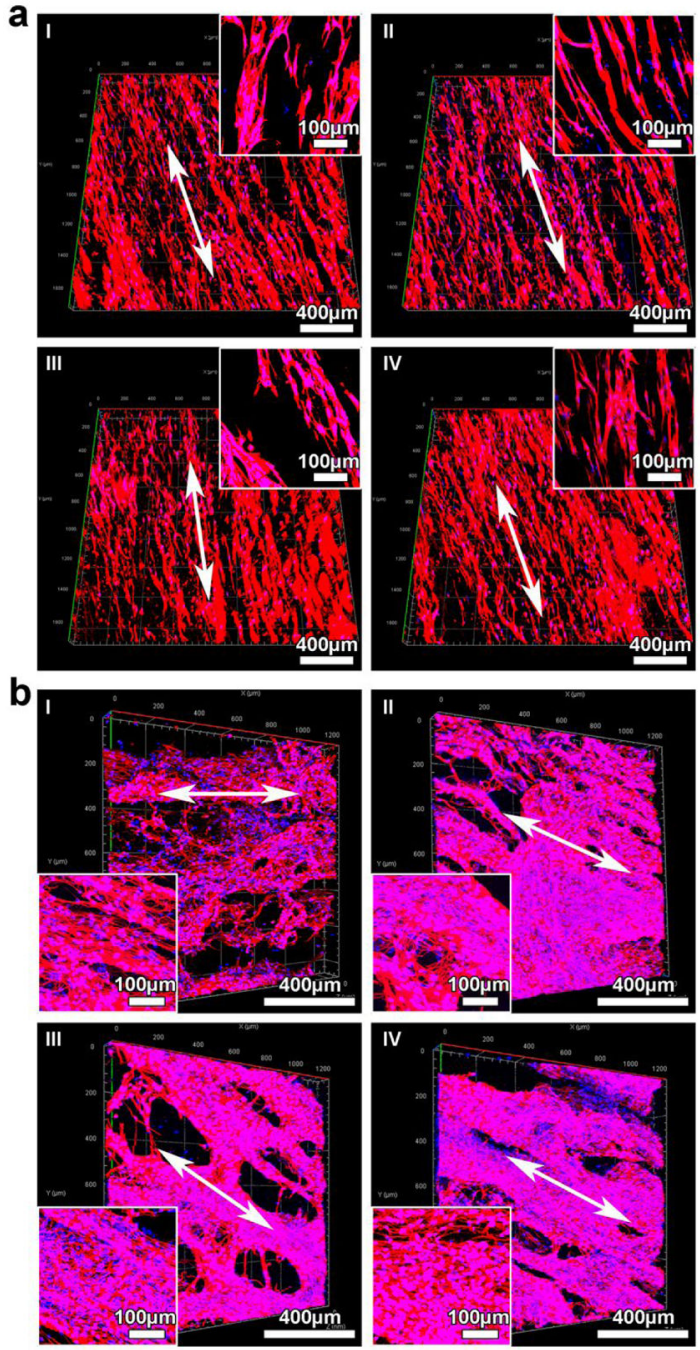

Figure 4.

Cell culture on 3D expanded nanofiber scaffolds $(2 \mathrm{~cm} \times 2 \mathrm{~cm} \times 1.5 \mathrm{~mm})$. (a) Conformal microscopy images showing BMSCs in different regions of square-shaped nanofiber scaffolds. The depth imaged was $125 \mu \mathrm{m}$. Inset: highly magnified images of BMSCs seeded on nanofiber scaffolds. Cells were stained with Alexa Fluor ${ }^{\mathrm{TM}} 546$ Phalloidin in red, and cell nuclei were stained with DAPI in blue. (b) Conformal microscopy images showing hNSCs in different regions of square-shaped nanofiber scaffolds after proliferation for 7 days and neuronal differentiation for another 14 days. Inset: The formation of 3D neuronal networks on the scaffolds after neuronal differentiation for 14 days. Cells were stained with Tuj 1 for neurons in red, and cell nuclei were stained with DAPI in blue. 

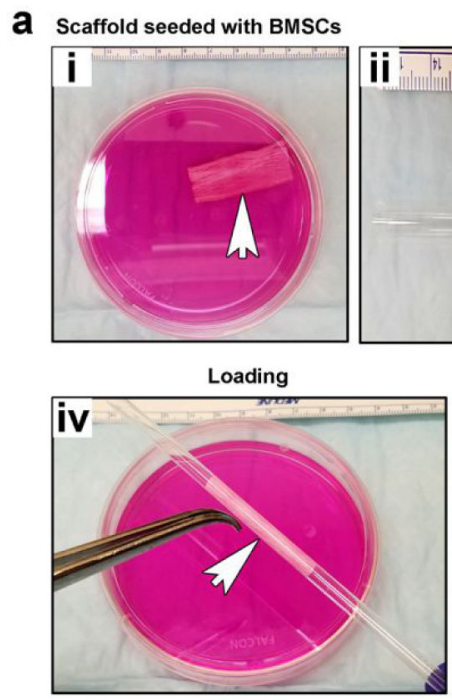

b

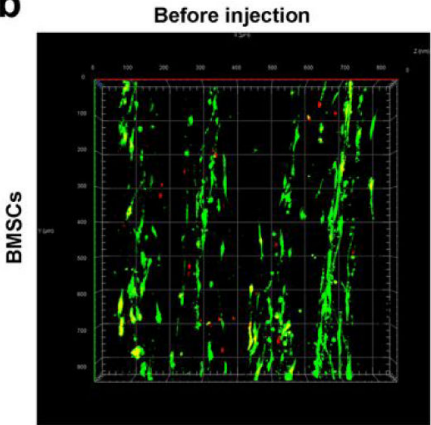

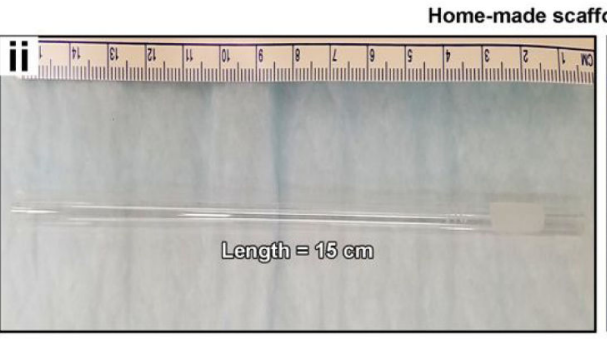

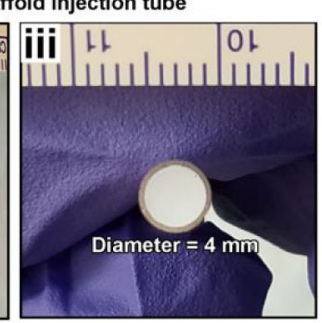

Re-expansion after injection
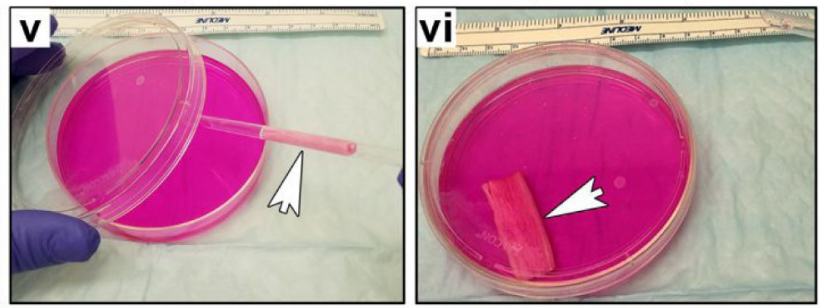

C
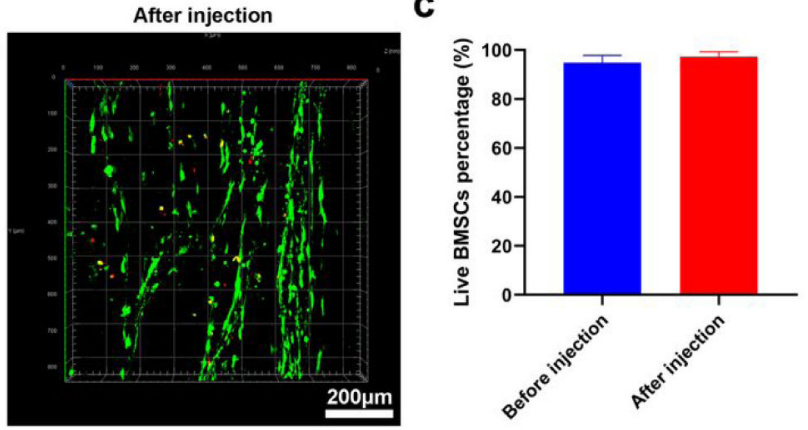

Figure 5.

Demonstration of minimally invasive delivery of 3D tissue constructs and the effect of delivery on the cell viability. (a (i)) Photograph showing a 3D tissue construct consisting of $0.05 \%$ GelMA-coated, 3D expanded nanofiber scaffolds seeded with BMSCs in a petri dish. (a (ii, iii)) Photographs showing glass tubes for injection of 3D tissue constructs. (a (iv, v)) Photographs showing the 3D tissue construct was loaded into the glass tube and then injected into the petri dish containing culture medium using an air source. (a (vi)) Photograph showing the injected 3D tissue construct returned to its original shape. (b) LIVE/ DEAD staining of BMSCs seeded on $0.05 \%$ GelMA-coated, 3D expanded nanofiber scaffolds before and after injection. (c) Quantification of live BMSCs before and after injection. 